\title{
Neuroimaging of intrinsic connectivity networks: a robust method for assessing functional brain organization in psychiatric disorders
}

\author{
Amy S. Garrett, (iD Steven R. Pliszka \\ Department of Psychiatry, University of Texas Health Science Center, San Antonio, TX, USA.
}

Neuroimaging scientists are well aware that even simple cognitive processes require contributions from multiple brain regions working together as a network. Now, computational tools to identify and assess these networks are readily available, and a growing number of studies are showing that connectivity within and between networks provides important measures of psychiatric disorders.

Functional connectivity is a measure of the temporal synchronization of activity in spatially separate brain regions, suggesting that these regions are working together as a network. It is easy to conceptualize how brain regions would be synchronized while performing a task. For example, while following a visual target, it makes sense that activity in the visual cortex would be synchronized with activity in the frontal eye fields, as both of these regions are needed to perform this task. However, it is not intuitively obvious that neural activity is synchronized while not performing a task. Biswal et al. were the first to report that low frequency oscillations in the left and right sensorimotor cortices are temporally synchronized while subjects rest in the scanner. ${ }^{1}$ This finding implies that the brain maintains a network organization regardless of current demands, an effect called "intrinsic connectivity" referring to coordinated activity that is inherent to brain function rather than elicited by a task. Examples of commonly discussed intrinsic connectivity networks include the default mode network, the salience network, and the executive control network.

Although they are not fully understood, many scientists believe that intrinsic connectivity networks represent the functional organization of the brain that developed through frequent co-activation in the service of behavior, e.g. the Hebbian theory that "neurons wire together if they fire together."2 Notably, resting state intrinsic connectivity is based on low-frequency oscillations (0.01-0.1 hertz), while task-based connectivity is based on a higher frequency signal ( $\sim 0.5$ hertz). However, the two measures are spatially similar. A meta-analysis of functional magnetic resonance imaging (fMRI) data from nearly 40,000 subjects showed a strong concordance between spatial patterns of task activation and 20 of the most

Correspondence: Amy S. Garrett

E-mail: garrettas@uthscsa.edu reliably observed intrinsic networks. ${ }^{3}$ For example, the executive control network is similar to patterns of activation during cognitive tasks and the salience network aligns with emotional tasks. For these reasons, we may conclude that aberrant intrinsic connectivity indicates abnormal brain organization and could be used to better understand the neural correlates of psychiatric disorders.

In addition to connectivity within intrinsic networks, connectivity between networks is a meaningful metric for studies in psychiatry. Connectivity between networks indicates the ways in which these networks interact, for example, in healthy people, the default mode network has a negative connectivity with the dorsal attention network to effectively transition attention from the internallyfocused default mode to the externally-focused attention network. In contrast, youth with attention deficit hyperactivity disorder (ADHD) show hyperconnectivity between these networks, consistent with symptoms of dysregulated attention. ${ }^{4}$ Furthermore, brain development during adolescence is characterized by increasing connectivity between networks and decreasing connectivity within networks, ${ }^{5}$ providing a metric by which aberrant or delayed neural development can be assessed.

In the current issue, Battel et al. ${ }^{6}$ successfully identify 3 important intrinsic connectivity networks in a group of 29 adolescents with depression, providing a view of functional brain organization that is consistent with previous studies in adolescents. The consistency of these results is an important strength of resting state connectivity methods, and the work reported here is meaningful in that it contributes one of the first fMRI studies of youth with depression in a middle-income country.

Resting-state fMRI has advantages over task-based $\mathrm{fMRI}$ in the study of clinical disorders. Patient groups who cannot comply with task demands (such as those with severe ADHD or autism) can participate, language differences between subject groups are not an issue, and variability in task design does not lead to difficulties in comparing results from different studies. Using resting state fMRI data, large normative databases of brain connectivity can be developed that may ultimately lead to the

How to cite this article: Garrett AS, Pliszka SR. Neuroimaging of intrinsic connectivity networks: a robust method for assessing functional brain organization in psychiatric disorders. Braz $\mathrm{J}$ Psychiatry. 2020;42:1-2. http://dx.doi.org/10.1590/1516-4446-20200002 
establishment of clinical standards for assessing aberrant connectivity for an individual patient.

How could resting-state intrinsic connectivity information be used clinically? Clearly, we do not need a functional MRI scan to determine if someone is depressed or has ADHD. However, with further research, several scenarios could become possible. For example, it could be possible to subtype individuals with a disorder based on their intrinsic connectivity. Such subtypes could indicate likelihood to respond to a particular antidepressant or suggest that an individual is a better candidate for psychotherapy. Alternately, a particular intrinsic connectivity pattern may indicate that more aggressive treatment is needed. Ultimately, clinical applications developed from MRI data could be translated to less expensive forms of measurement, such as functional near infrared spectroscopy or electroencephalogram. The investigation reported by Battel et al. ${ }^{6}$ brings this day closer.

\section{Disclosure}

SRP receives royalties from Guilford Press, is a consultant for the State of Texas, and does contracted research for Ironshore Pharmaceuticals and Shire. The other author reports no conflicts of interest.

\section{References}

1 Biswal B, Yetkin FZ, Haughton VM, Hyde JS. Functional connectivity in the motor cortex of resting human brain using echo-planar MRI. Magn Reson Med. 1995;34:537-41.

$2 \mathrm{Hebb}$ DO. The organization of behavior; a neuropsychological theory. A Wiley book in clinical psychology. New York: Wiley; 1949.

3 Smith SM, Fox PT, Miller KL, Glahn DC, Fox PM, Mackay CE, et al. Correspondence of the brain's functional architecture during activation and rest. Proc Natl Acad Sci U S A. 2009;106:13040-5.

4 Lin $H$, Lin $Q$, Li $H$, Wang $M$, Chen $H$, Liang $Y$, et al. Functional connectivity of attention-related networks in drug-naive children with ADHD. J Atten Disord. 2018 Sep 27: 1087054718802017. doi: 10.1177/1087054718802017. [Epub ahead of print]

5 Marek S, Hwang K, Foran W, Hallquist MN, Luna B. The contribution of network organization and integration to the development of cognitive control. PLoS Biol. 2015;13:e1002328.

6 Battel L, Swartz J, Anes M, Manfro PH, Rohde LA, Viduani A, et al. Neuroimaging adolescents with depression in a middle-income country: feasibility of an fMRI protocol and preliminary results. Braz $\mathrm{J}$ Psychiatry. 2020;42:6-13. 